\title{
Phase-Space Reconstruction: A Path Towards the Next Generation of Nonlinear Differential Equation Based Models and Its Implications Towards Non-Uniform Sampling Theory
}

The INL is a

U.S. Department of Energy

National Laboratory

operated by

Battelle Energy Alliance

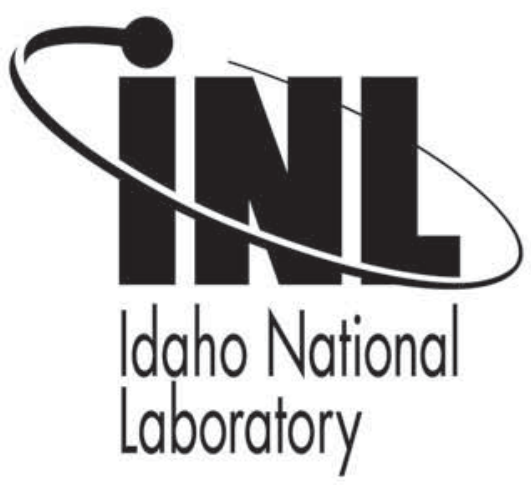

\section{ISRCS 2009 2nd International Symposium on Resilient Control Systems}

Charles R. Tolle

Mark Pengitore

August 2009

This is a preprint of a paper intended for publication in a journal or proceedings. Since changes may be made before publication, this preprint should not be cited or reproduced without permission of the author. This document was prepared as an account of work sponsored by an agency of the United States Government. Neither the United States Government nor any agency thereof, or any of their employees, makes any warranty, expressed or implied, or assumes any legal liability or responsibility for any third party's use, or the results of such use, of any information, apparatus, product or process disclosed in this report, or represents that its use by such third party would not infringe privately owned rights. The views expressed in this paper are not necessarily those of the United States Government or the sponsoring agency. 


\section{Phase-Space Reconstruction: a Path Towards the Next Generation of Nonlinear Differential Equation Based Models and Its Implications Towards Non-Uniform Sampling Theory}

\author{
Charles R. Tolle \\ South Dakota School of Mines and Technology \\ Electrical and Computer Engineering Department \\ Rapid City, SD 57701 USA \\ Email: Charles.Tolle@sdsmt.edu
}

\author{
Mark Pengitore \\ South Dakota School of Mines and Technology \\ Mathematics and Computer Science Department \\ Rapid City, SD 57701 USA
}

\begin{abstract}
This paper explores the overlaps between the Control community's work on System Identification (SysID) and the Physics, Mathematics, Chaos, and Complexity communities' work on phase-space reconstruction via time-delay embedding. There are numerous overlaps between the goals of each community. Nevertheless, the Controls community can gain new insight as well as some new very powerful tools for SysID from the latest developments within the Physics, Mathematics, Chaos, and Complexity communities. These insights are gained via the work on phase-space reconstruction of non-linear dynamics. New methods for discovering non-linear differential based equations that evolved from embedding operations can shed new light on hybrid-systems theory, Nyquest-Shannon's Theories, and network based control theory. This paper strives to guide the Controls community towards a closer inspection of the tools and additional insights being developed within the Physics, Mathematics, Chaos, and Complexity communities for discovery of system dynamics, the first step in control system development. The paper introduces the concepts of phase-space reconstruction via time-delay embedding (made famous by Whitney, Takens, and Sauer's Thoreoms), intergrate-and-fire embedding, and non-linear differential equation discovery based on Perona's method.
\end{abstract}

\section{INTRODUCTION}

The core of control system technology is modeling. The reason for this is that before one can attempt to implement any of the key control system technologies, one must first have a useful model for that system. It is no surprise that as one improves the model of a system, the control of that system can also be improved. Today, the gold standard for models is a non-linear differential equation based

This work was in part supported by the Idaho National Laboratory's (INL's) Laboratory Directed Research and Development (LDRD) Program under DOE Idaho Operations Office Contract DE-AC07-05ID14517. Approved for external release, STI Number: INL/CON-09-16271 expression for the system to be controlled. The majority of all control technologies first begin with this type of model. This is true even for linear based controllers, whose development standardly begins with the linearization of the non-linear differential equation based model.

Like the Controls community, the Physics, Mathematics, Chaos, and Complexity communities have also focused on non-linear differential equation based expressions to model their dynamical systems. Similar to the Controls community, these communities discuss observation theory and system identification using a vastly different nomenclature and language. Nevertheless, these communities are working on the cutting edge of nonlinear dynamics and observation of those dynamics. Much of their work remains inaccessible and unused within the general Controls community. It is for that reason, that this paper introduces the concepts of phase-space reconstruction via time-delay embedding (made famous by Whitney[1], Takens[2], and Sauer's[3] Theorems), integrate-and-fire embedding[4],[5], and non-linear differential equation discovery based on Perona's method[6],[7]. A concise and accessible overview of the embedding and phase-space reconstruction methods is given in [8]. It is the focus of this paper to tie these concepts together in the light of control theory, and thus begin to chart a new path forward towards an automated method for nonlinear differential equation creation, i.e. automated first principles like modeling. Such a method, when 
fully developed, has a vast outlet of uses: e.g. the automated discovery of dynamics can be used to track on-going changes within a plant's fundamental dynamics; which in turn, can be interpreted to predict on-coming failures (prognostication and fault detection). In addition, by automatically discovering changes in a non-linear plant on the fly, one might be able to develop a new class of robust adaptive controllers that can validate close-loop stability assumptions online to allow for graceful shutdown of the plant before catastrophic failure can occur. This would be achieved by monitoring the plant as it drifts away from the nominal predicted operating condition, the baseline non-linear model. This new type of controller can re-evaluate the validity of its control strategy, and take appropriate action before the assumptions become invalid, which could lead to catastrophic failure of a system. The tools being currently developed in [6] and [7] are the basis of this new approach (the first implementation step). In order to see how this might be developed, one first needs to understand how phase-space reconstruction relates to control theory and how it can play a part in the development of a new control system design and implementation method.

\section{OBSERVERS AND PHASE-SPACE RECONSTRUCTION}

For some time, it has been known that by sampling a portion of a system's states, one can reconstruct missing or unmeasurable states needed for the construction, and implementation of full state feedback control. Within the controls community such a process/algorithm is known as an observer. There are many types of these observers, e.g. the Luenberger observer [9], [10], [11], [12], Kalman Filters[9], [13], [14], and extended Kalman Filters[15]. Of course, the Kalman filter is the optimal observer given a linear system disturbed by a gaussian white noise process. However, in this paper we are interested in non-linear systems as well. The extended Kalman filter can address such systems, but it is no longer optimal and is prone to instabilities. Each of these estimators, when properly used and designed, can estimate the states of a system in real time. In some sense, this is a far cry from discovering dynamics. Alternately, there are a number of least square approaches that can simply estimate a system's dynamics on the fly instead of estimating the states directly. Such systems like auto-regessive moving-average (ARMA) [16], [17] estimators use the concepts of phasespace reconstruction within their design.

Consider a linear plant model defined as:

$$
\begin{aligned}
& \dot{x}(t)=A x(t)+B u(t) \\
& y(t)=C x(t)+D u(t)
\end{aligned}
$$

where $x, y, u$ are defined as: $x(t)=$ $\left[\begin{array}{llll}x_{1}(t) & x_{2}(t) & \ldots x_{k}(t)\end{array}\right]^{\prime}$ (the internal states), $y(t)=\left[\begin{array}{llll}y_{1}(t) & y_{2}(t) & \ldots & y_{l}(t)\end{array}\right]^{\prime}$ (the observable features), and $u(t)=\left[\begin{array}{lll}u_{1}(t) & u_{2}(t) \ldots u_{m}(t)\end{array}\right]^{\prime}$ (the controls). One can define a state estimator, $\dot{\hat{x}}(t)$, as:

$$
\dot{\hat{x}}(t)=A \hat{x}(t)+B u(t)+K(y(t)-C \hat{x}(t))
$$

where the choice of $K$ controls the estimators means and rate of convergence. Of course, it has been shown that $\lim _{t \rightarrow \infty} \dot{\hat{x}}(t)=\dot{x}(t)$, given that certain conditions on the system exist; such as, the observability matrix $(O=$ $\left.\left[C^{\prime} A^{\prime} C^{\prime} \ldots\left(A^{\prime}\right)^{k-1} C^{\prime}\right]\right)$ must be of full rank, i.e. $\operatorname{rank}(O)=k$. There exists a direct parallel to these ideas within the phase-space reconstruction methods.

To begin, phase-space reconstruction was not developed with the limitation of linear systems theory. In fact, phase-space reconstruction works with both discrete time maps as well as continuous time based differential equations[8]. Consider a system of nonlinear first-order differential equations of the form:

$$
\dot{\bar{x}}_{i}(t)=\sum_{k=1}^{S} a_{i, k} \bar{A}_{i, k}(\bar{x}(t))+\sum_{j=1}^{I} b_{i, j} \bar{B}_{i, j}(\bar{u}(t))
$$

where $S$ and $I$ are the number of allowed basis functions for the internal feedback and forcing dynamics within the system respectively, and the $a_{i, k}$ and $b_{i, j}$ are constants controlling the contribution of that basis function to the state, $\bar{x}_{i}$. Given this 
framework, one might consider any type of linear or nonlinear basis functions, e.g. $\bar{A}_{i, k}(\bar{x}(t))=\bar{x}_{1}(t)$ or $\bar{A}_{i, k}(\bar{x}(t))=\bar{x}_{1}(t) \bar{x}_{2}^{2}(t)$ or even $\bar{A}_{i, k}(\bar{x}(t))=$ $\operatorname{sign}\left(\bar{x}_{1}(t) \bar{x}_{2}(t)\right)$. Note, that the basis functions allowed in eqn. 3 are assumed to be a super-set of the actual dynamics. There does not currently exist a means to guaranty if the basis contains the proper set of functions. The only current means to check this is by re-evolving the dynamics once the fit is complete and verifying that the system in fact reproduces similar dynamics as the original embedded space. The actual process used to reconstruct the phase-space is known as time-delay embedding. The original theorems are based on the system of nonlinear dynamics being unforced, i.e. $b_{i, j}=0$. Casdagli et. al. has extended the embedding idea to forced dynamical systems[18], i.e. $b_{i, j} \neq 0$. Time-delay embedding is accomplished by measuring successive-time-delayed values from a fixed linear combination of the system's original state variables. In general, the time-delay, $\tau$, maybe arbitrary (although it is believed that by choosing $\tau$ appropriately, less distortion is introduced into the phase-space reconstruction[19]). For an unforced system, the time embedment is defined as:

$$
\hat{\bar{x}}(t)=[W \bar{x}(t) W \bar{x}(t+\tau) \ldots W \bar{x}(t+2 k \tau)]
$$

where $W$ is a row matrix defining an arbitrary linear combination of the original state variables. Whitney[1], Takens[2], and Sauer's[3] theorems state that this embedment is a diffeomorphic representation of a system's original phase space. Gershenfeld describes this in the most accessible way using the following analogy: consider a 2dimensional phase-space existing on a rubber sheet of paper. By performing a time-delay embedment of successive measurements, one is at most simply stretching and twisting the original phase space within a higher dimensional space[8]. In fact, depending on which theorem one uses (e.g. what constraints are placed on the system - similar to the full rank condition within the observer formulation) the most additional dimensions that one might twist the phase space into is $2 D+1$, where $D$ is the original dimension of the systems phasespace. Nevertheless, there remains an analog to the full rank condition in linear systems theory. This analog is simply that the system states must be fully connected, i.e. if one state changes those effects can be seen in each of the other states. This is simply stating in words the full rank condition within linear systems theory for a nonlinear system. In essence, the embedding theorems give us a transformed phase-space:

$$
\hat{\bar{x}}(t)=V_{\text {locally }} \bar{x}(t)
$$

where $V$ defines a smooth local linear transformation matrix over a local region, i.e. one-to-one holds but not necessarily onto. The full space can exist as a single or multiple localized series of these smooth transformations. This is a bit of a stretch in nomenclature but it gets the idea across. From an engineering and SysID perspective the lack of onto can be ignored, i.e. if we make successive timedelayed measurements of our system and embed them to reconstruct the original phase-space, our reconstructed phase-space will only contain points that were transformed from the original space. Thus if one uses these measured points to help discover the quote unwrapped dynamics - the points will have a inverse mapping (one-to-one).

Discovering phase-space or even a wrapped representation of phase-space is nice in that it makes for nice pictures; but in controls, we need the differential equations that underlie that phase-space. Over the last two and half years, we have been developing a Matlab application that implements Perona et. al.'s method[6] in order to find the nonlinear differential equations that underlie a set of phase-space points. We have also studied the effects of noise on said method[7]. Currently, we are using Casdagli et. al.'s ideas[18] to extend the nonlinear differential equation reconstruction process to forced dynamical systems. In doing so we are replacing our original cost function[7]:

$$
\begin{gathered}
Q_{2}=\frac{1}{2} \sum_{j=1}^{j_{\max }} \sum_{l=1}^{l_{\max }} \sum_{i=1}^{D}\left[\sum_{z=1}^{K}\left(a_{i, z} \int_{t_{j}}^{t_{j}+d t_{l}} A_{i, z}(\tau) d \tau\right)\right. \\
\left.+\hat{x}_{i}\left(t_{j}\right)-x_{i}\left(t_{j}+d t_{l}\right)\right]^{2} .
\end{gathered}
$$


with an augmented cost function which includes the forcing terms:

$$
\begin{aligned}
Q_{3}= & \frac{1}{2} \sum_{j=1}^{j_{\max }} \sum_{l=1}^{l_{\max }} \sum_{i=1}^{D}\left[\sum_{z=1}^{K}\left(a_{i, z} \int_{t_{j}}^{t_{j}+d t_{l}} A_{i, z}(\tau) d \tau\right)\right. \\
& +\sum_{w=1}^{C} b_{i, w}\left(\int_{t_{j}}^{t_{j}+d t_{l}} B_{i, w}(\tau) d \tau\right)+\hat{x}_{i} \\
& \left.-x_{i}\left(t_{j}+d t_{l}\right)\right]^{2}
\end{aligned}
$$

Once one is able to discover the underling nonlinear differential equations that generate a particular phase-space portrait, one can use this method to proceed to implement the generalized embedding process to reconstruct unknown system phasespaces and thereby discover the nonlinear differential equations that underlie that particular phasespace portrait. Note in general the equations obtained from such a process are not as clean as those created during a first principle modeling process. Research on how to reduce the cross terms, e.g. $\bar{A}_{i, k}(\bar{x}(t))=\bar{x}_{1}(t) \bar{x}_{2}(t)$, by rotating and scaling the discovered equations to a more simplified form is needed. Note, additional cross terms arise in a first principle model by rotating and scaling the phase-space away from its primary basis vectors. Such a rotation and scale transformation is what occurs when one reconstructs the phase-space via the embedding process. Reversing these effects is what allows for the discovery of the first principle like model.

Even with the advancements in non-linear differential equation based SysID discussed above, yet more powerful versions of the embedding theorems can be brought to bare that might allow for discovery of event based dynamical systems such as welding detachments, dripping faucets, avalanches, etc. These extended methods are known as the integrateand-fire version of the embedding theorems[4], [5]. Under this extension the dynamical system is sampled using a "integrate-and-fire" process. Consider measuring a linear combination of state variable as before, but this time integrate that measurement up to a threshold value, $\theta$ :

$$
\theta=\int_{t_{n}}^{t_{n+1}} W \bar{x}(t) d t
$$

The choice of a threshold value, $\theta$, is key in the process. If the value is too great the dynamics are lost; while if the value is too small - the dynamics 7) become greatly deformed, just like the choice of $\tau$ in time-delay embedding. As before, by using the time-delays between each firing event one can again reconstruct the desired phase-space[4], [5]:

$\hat{\breve{x}}(t)=\left[\left(t_{n+1}-t_{n}\right)\left(t_{n+2}-t_{n+1}\right) \ldots\left(t_{n+2 k+1}-t_{n+2 k}\right)\right]^{\prime}$

Note the results to date are very noisy. Nevertheless, using this extended type of embedding, we should obtain a similar result as before:

$$
\hat{\breve{x}}(t)=V_{\text {locally }} \bar{x}(t)
$$

The full proof of this extension theorem has not been completed as of yet; this is due to lack of interest by the community, i.e. the usefulness of the initial embedding theorems have not been exploited to their fullest as is being laid out in this paper, so Sauer has not deemed it necessary to complete the extension theorem. It is hoped that by fully exploiting the initial theorem, Sauer and his associates will find it necessary to complete this proof. Consider the implications of the integrate-and-fire theorem to the engineering community. Where Nyquest places a limit on the minimum sample time required to avoid aliasing, the value $\theta$ relates a threshold limit on phase-space distance to time. Also, the timedelays themselves are enough to reconstruct phasespace and thus dynamics. As before once one has a portrait of phase-space one can use the differential reconstruction process developed by [6] and [7] to obtain the desired nonlinear differential equation needed to start the control system development process or a prognostication sub-system. Furthermore, consider the vast number of currently un-modeled systems that relate to integrate-and-fire dynamics, e.g. earthquakes, avalanches, etc. These types of systems are ubiquitous, however they remain largely 
unstudied due to modeling and conceptual understanding based difficulties.

The integrate-and-fire theorem might also shed new insight into the process of how one should choose the optimal time-delay $\tau$ when embedding is performed. As was discussed above, by varying $\tau$, one changes the amount of stretching and twisting that is introduced into the phase-space reconstruction. Such an effect implies that there might be a particular $\tau$ that minimizes any distortions introduced into the reconstruction process. Traditionally, $\tau$ has been chosen using mutual information theory[20], [19]. Moreover, in discussions with Sauer about what choice might be used for $\tau$, he stated that there was no particular requirement on choosing a fixed time-delay between successive embedment terms, as shown in eqn. 4 . The only real requirement is that one doesn't continuously sample only one region of the phase-space. One must choose a $\tau$ that allows one to visit the complete space of interest. This directly opens the concept of non-uniform sampling of the embedding vector in eqn. 4. It is clear that from a dynamics perspective, time and phase-space evolution change as you move about within it. Perhaps this suggests that a fixed sample time is not always appropriate, e.g. aircraft dynamics are generally broken into two separate models which represent the long period and short period dynamics. Consider Fig. 1, the grey lines represent trajectories within an exampled phasespace, while the dots along those lines represent equal distance steps for a fixed time-delay.

Clearly as one approaches a fixed point, the movement within the phase-space slows, i.e. the dynamics slow. This slowing is shown in Fig. 1 as smaller steps along the trajectory for a fixed time step near the origin. Likewise, as one moves away from the fixed point the dynamics increase in speed. One might conclude that depending on what part of phase-space one is in, one might choose different $\tau \mathrm{s}$. This introduces the notion of optimal sample time or perhaps non-uniform sample time. To date this remains an open problem. One possible step towards a solution is Time-Based Clustering (TBC) [21], [22]. TBC solves an optimization problem for which Fraser's mutual information estimator ap-

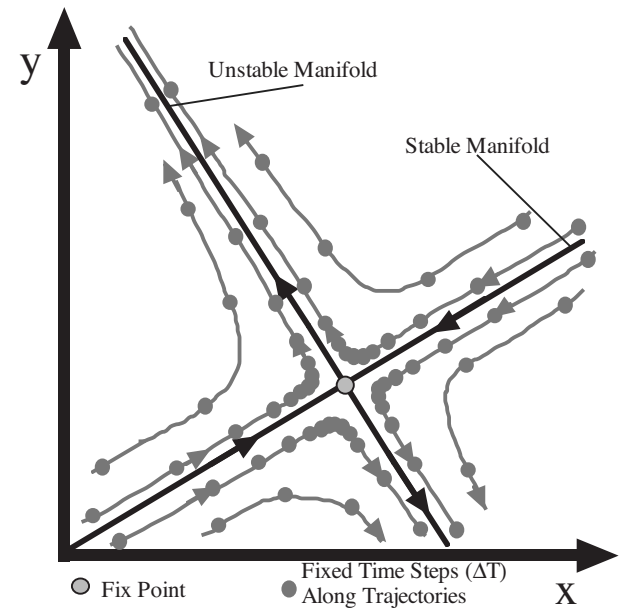

Fig. 1. This figure shows an example phase-space, which demonstrates the nonuniform nature to fixed time-delays verses phase-space distance traveled.

pears as a special case[21]. TBC might also be used to set the delay-times in eqn. 4 at different intervals. TBC might also be introduced into the equation reconstruction process to optimize the sample delays used when choosing the comparison points within the optimization process in [7]. Currently, when using a fixed time-delay (or sample time), one forces any optimization problem or knowledge discovery process to favor portions of the phase-space, which are slowly evolving, i.e. one obtains more samples within that region. In addition to TBC, calculus of time scales is also a fast developing field that might shed further insight in to sampling theory and the spectrum of hybrid systems that exist between discrete time dynamics and continuous time dynamics [23], [24]. These concepts of varying times delays to optimally understand dynamics has interesting ramifications on the engineering world and on distributed hybrid-network control systems.

\section{CONCLUSION}

By extending Perona et. al.'s method[6] to forced dynamical systems, a new method of nonlinear SysID can be developed based on direct phasespace reconstruction. Currently, we have extended our cost function in [7] (eqn. 6) to include forcing dynamics (eqn. 7). The linear algebraic equations 
needed to solve the optimization problem defined by this extension, eqn. 7, have been completed. We are in the process of updating our Matlab program to solve the extended problem. In the out years, we plan on testing these methods on partial differential equations, as well as developing a method to reduce cross terms obtained via the phase-space embedding process. In addition, using the equation based reconstruction process; one should be able to develop a numerical example of the embedding theorems. This has yet to be done due to the difficulties in finding a mapping function between two nonlinear spaces. Furthermore, the effects and means to incorporate TBC into the phase-space reconstruction process as well as the equation reconstruction method need further investigation. Finally, more thought is needed to fully comprehend the ramifications of the integrate-and-fire embedding conjecture and what the relationship of a threshold on phase-space distance to dynamics means in the face of sampling theory and reconstructability.

\section{ACKNOWLEDGMENT}

The authors would like to thank the Idaho National Laboratory's Instrument Controls and Intelligent Systems (ICIS) Signature for supporting this project. Moreover, Mr. Pengitore thanks the Rocky Mountain NASA Space Grant headquartered at Utah State University, Logan, Utah for supporting his efforts on this project. In addition, we thank Laura J. Tolle for her careful editing of this paper.

\section{REFERENCES}

[1] H. Whitney, "Differentiable manifolds," Ann. of Math., vol. 37, pp. 645-680, 1936.

[2] L. Noakes, "The takens embedding theorem," International Journal of Bifurcation and Chaos, vol. 1, no. 4, pp. 867872, 1991.

[3] T. Sauer, J. A. Yorke, and M. Casdagli, "Embedology," Journal of Statistical Physics, vol. 65, no. 3/4, pp. 579616, 1991

[4] T. Sauer, "Reconstruction of dynamical systems from interspike intervals," Phys. Rev. Lett., vol. 72, no. 24, pp. 3811-3814, Jun 1994.

[5] - - , "Reconstruction of integrate-and-fire dynamics," American Mathematical Society, pp. 63-75, 1996.

[6] P. Perona, A. Porporato, and L. Ridolfi, "On the trajectory method for the reconstruction of differential equations from time series," Nonlinear Dynamics, vol. 23, no. 1, 2000.

[7] K. H. Warnick, C. R. Tolle, and J. W. James, "Exploration of the trajectory method for reconstructing nonlinear differential equations from time series," Physica D, vol. Currently under review., 2007.
[8] N. Gershenfeld, The Nature of Mathematical Modeling, 6th ed. The Edinburgh Building, Cambridge CB2 2RU, UK: Cambridge University Press, 1999.

[9] B. Friedland, Control System Design: An Introduction to State-Space Methods, ser. McGraw-Hill Series in Electrical Engineering, S. W. Director, Ed. New York, NY: McGrawHill, Inc., 1986.

[10] D. G. Luenberger, "Obsevering the state of a linear system," IEEE Trans. on Military Electronics, vol. MIL-8, pp. 74-80, April 1964

[11] - -, "Obsevers for multivariable systems," IEEE Trans. on Automatic Control, vol. AC-11, no. 2, pp. 190-197, April 1966.

[12] - - "An introduction to observers," IEEE Trans. on Automatic Control, vol. AC-16, no. 6, pp. 596-602, December 1971.

[13] R. E. Kalman, "A new approach to linear filtering and prediction problems," Trans. ASME (Jounrnal Basic Engineering), vol. 82D, no. 1, pp. 35-45, March 1960.

[14] R. E. Kalman and R. S. Bucy, "New results in linear filtering and prediction theory," Trans. ASME (Jounrnal Basic Engineering), vol. 83D, no. 1, pp. 95-108, March 1961.

[15] F. Orderud, "Comparison of kalman filter estimation approaches for state space models with nonlinear measurements," in Proceedings of Scandinavian Conference on Simulation and Modeling. http://www.scansims.org/: SIMS (Scandinavian Simulation Society), Oct. 2005.

[16] G. C. Goodwin and K. S. Sin, Adaptive Filtering Prediction And Control, ser. Information and System Sciences Series, T. Kailath, Ed. Lodon, UK: Prentice-Hall International, Inc., 1984.

[17] D. G. Luenberger, Linear and Nonlinear Programming, 2nd ed. Addison-Wesley Publishing Co., Inc., May 1989.

[18] M. Casdagli, "A dynamical systems approach to modeling input-output systems," in Nonlinear Modeling and Forecasting, ser. SFI Studies in the Sciences of Complexity, M. Casdagli and S. Eubank, Eds., vol. XII, Santa Fe Institute. Redwood City, CA: Addison-Wesley, 1992, pp. 265-281.

[19] H. D. I. Abarbanel, Analysis of Observed Chaotic Data. New York: Springer-Verlag, 1996.

[20] A. M. Fraser and H. L. Swinney, "Independent coordinates for strange attractors from mutual information," Phys. Rev. A, vol. 33, no. 2, pp. 1134-1140, 1986.

[21] C. R. Tolle, "Time-based clustering and its application to determining a signal's motivation: Deterministic chaos or random disturbance," Ph.D. dissertation, Utah State University, 1997.

[22] C. R. Tolle and R. W. Gundersen, "Searching for determinism in erratic signals: Fuzzy time-based clustering of delay vectors," in Proceedings of ANNIE 1998 (Intelligent Engineering Systems Through Artificial Neural Networks), 1-4 November 1998, St. Louis, Missouri, USA, vol. 8. ASME, Nov. 1998, pp. 43-48.

[23] M. Bohner and A. Peterson, Dynamic Equations on Time Scales: An Introduction with Applications. Boston, Massachusetts: Birkhauser, 2001.

[24] M. Bohner and A. Peterson, Eds., Advances in Dynamic Equations on Time Scales. Birkhauser, 2003. 